\title{
Research on the Design of Government Affairs Platform in the Context of Big Data
}

\author{
Qian Huang $\mathbb{D}^{1,2}$ and Xue Wen $\mathrm{Li}^{3}$ \\ ${ }^{1}$ School of Information Management Wuhan University, Wuhan 430072, China \\ ${ }^{2}$ School of Marxism North Minzu University, Yinchuan 750021, China \\ ${ }^{3}$ North Minzu University Library, Yinchuan 750021, China \\ Correspondence should be addressed to Qian Huang; huangqian110@whu.edu.cn
}

Received 26 March 2021; Revised 26 April 2021; Accepted 5 May 2021; Published 30 May 2021

Academic Editor: Shah Nazir

Copyright (C) 2021 Qian Huang and Xue Wen Li. This is an open access article distributed under the Creative Commons Attribution License, which permits unrestricted use, distribution, and reproduction in any medium, provided the original work is properly cited.

\begin{abstract}
Big data is a massive and diverse form of unstructured data, which needs proper analysis and management. It is another great technological revolution after the Internet, the Internet of Things, and cloud computing. This paper firstly studies the related concepts and basic theories as the origin of research. Secondly, it analyzes in depth the problems and challenges faced by Chinese government management under the impact of big data. Again, we explore the opportunities that big data brings to government management in terms of management efficiency, administrative capacity, and public services and believe that governments should seize opportunities to make changes. Brainlike computing attempts to simulate the structure and information processing process of biological neural network. This paper firstly analyzes the development status of e-government at home and abroad, studies the service-oriented architecture (SOA) and web services technology, deeply studies the e-government and SOA theory, and discusses this based on the development status of e-government in a certain region. Then, the deep learning algorithm is used to construct the monitoring platform to monitor the government behavior in real time, and the deep learning algorithm is used to conduct indepth mining to analyze the government's intention behavior.
\end{abstract}

\section{Introduction}

Government management is bound to have major reforms and innovations under the help of big data. It will not only enhance the government's social management capabilities, but also improve the government's management level. However, the application of big data in China is still in a relatively basic development stage. The government will face many difficulties in applying big data for management and decision-making. It is necessary to clearly understand these issues before they can find breakthrough points one by one. The application of data in government management lays a solid foundation. Under the impact of big data, the scientific and technological progress stimulated by the massive data on the Internet will inevitably bring about tremendous changes in the way of life, thinking, and decision-making of human beings. Under these changes, the value of correctly using big data will be social life and economic development, which have far-reaching effects. First, big data revolutionizes the way humans think [1]. Big data changes the shift of human thinking patterns from causality to related relationships. Today's data in life is growing faster than ever before, and the amount of data is growing wildly. Brain-like computing seeks to design a system like biological neural network from the structure, seeks to simulate the function of the brain from the function, and seeks to greatly surpass the biological brain from the performance, also known as neuromorphic computing. The rapid update of information in the context of big data and the rapid changes in the environment require people to respond quickly when they encounter problems, rather than to explore the reasons. Second, big data revolutionizes the way humans make decisions.

In the past era of small data, human judgments on things and perceptions often rely on intuition and experience, and 
the resulting bias can be seen everywhere. The scientific predictions and decisions brought about by big data will inevitably change the way people make decisions. The significance of big data to decision makers lies in advance prediction, in-process perception, and after-the-fact feedback. People will be more dependent on data and analysis than on intuition and experience; likewise, it will change the nature of leadership and management. For the government, big data analysis has promoted the scientific decisionmaking of the government. Decision-making entities use big data to obtain real-time information in the decision-making process, master development trends, and make accurate predictions and scientific decisions. In resource allocation, big data also acts as the third hand. Big data has played a crucial role in driving decision-makers to change decisions and improve decision-making, and this is gradually rising to the national consciousness. Third, big data drives the modern transformation of government management. First, big data helps diversify management entities. In the era of small data, because of limited access to information, people are in an environment of information confinement, and these information resources are usually in the hands of some leaders, resulting in the public lack of understanding of information, and thus, they cannot effectively participate in management [2].

The arrival of the era of big data breaks the phenomenon of monopoly of information. People can obtain massive amounts of data information through various channels such as the Internet at all times, thus supervising the management activities of managers and making them more transparent and transparent. Big data also provides a channel for public participation. People can express their opinions through social networking sites at any time and express their needs. The public is an important factor in management activities, and big data helps achieve diversification of management entities. Second, big data facilitates the transformation of one-way management from collaborative shared management. In the past, different government departments had their own information systems, which caused many data to be uncommon and isolated from each other, forming an isolated island of information. Big data broke this barrier, realized data sharing, and maximized the effectiveness of data. It has improved the economic and social development and brought more convenience to the public. Finally, big data helps improve the scientific level of the decisionmaking process. In the past, management decision makers often used past experience to make decisions and judgments. Today's changes in the social environment and relationships often result in decision-making mistakes. Scientific decisionmaking under the foundation and guarantee of big data has become a trend of development. Decision makers are aware of the importance of data and use data for analysis and judgment to make scientific decisions [3]. The specific contributions of this paper include the following:

(i) The problems and challenges faced by Chinese government management under the impact of big data are deeply analyzed (ii) Deep learning algorithm is used to construct a monitoring platform for real-time monitoring of government behavior

(iii) The development status of e-government at home and abroad is analyzed

(iv) Using big data to update information quickly and making quick judgments

The rest of this paper is organized as follows. Section 2 discusses basic technology of SSEGA System Architecture, followed by construction of data platform based on brainlike computing in Section 3. SBEGA application in e-government platform is discussed in Section 4. Section 5 concludes the paper with summary and future research directions.

\section{Government Platform Architecture Based on SSEGA System}

Service-oriented architecture (SOA) was first proposed by Gartner in a 1996 report [4]. It is a service-centric, loosely coupled, dynamically optimized and reusable extended distributed applied constructor. After more than ten years of practice and development, it has become the trend of constructing integrated distributed information systems in an open and heterogeneous network environment. The loose coupling and interoperability of IT systems are brought by SOA [4], and the resulting large-scale reuse, large-scale integration, flexibility enhancement, etc. are the establishment, integration, operation, and maintenance of software systems. In particular, the innovation and development of the Internet-based software industry have brought new impetus and opportunities [5]. With the in-depth development of IT systems, the emergence of the "information island" problem and the rise of XML language and web services technology have objectively promoted the development of SOA. SOA has gradually entered people's careers and gradually shifted from concept to application. SOA requires a series of technical support, combined with web services and XML technology, to make the architecture more flexible and open; in the aspect of security authentication, SOAP can enhance interface access control and data security; in data storage, through distributed database processing, high concurrent requests can also dynamically add storage nodes. This chapter analyzes technologies such as service-oriented architecture technology and related network services and extended markup languages.

2.1. Basic Technology of SSEGA System Architecture. Combining the characteristics of SOA and web services technologies, it is necessary to change the current situation that the information between e-government systems of $\mathrm{B} / \mathrm{S}$ three-tier structure is difficult to share and interconnect, and use web services technology to encapsulate core services into open-access and remotely accessible services. It is a viable way to solve this problem. As an open service implementation specification, web services have been widely accepted by information system providers and IT technicians around the 
world and have gradually become the de facto standard for service implementation. [6].

Web services solve the problem of service implementation extremely well, but the emergence of services also challenges the traditional system architecture. The traditional $\mathrm{B} / \mathrm{S}$ three-tier structure cannot adapt well to service-oriented applications and must be from the system architecture. There is innovation at the level. ServiceOriented Architecture (SOA) was developed under such circumstances. Although SOA is still in the process of continuous improvement, it has been accepted and sought after by major platform providers and system providers. As described in Section 1, several of the world's largest IT vendors are launching their own SOA platforms and solutions, all of which provide the impetus for the overall development of SOA.

The problem now is that although everyone is pushing their own SOA platform and solutions, in actual project applications, SOA still mostly stays in the application of integration of legacy systems, and there is a lack of reference in the field of e-government, a common system architecture. Referring to the SOA architecture template proposed by IBM, this paper proposes SOA-Based e-Government Architecture (SBEGA), as shown in Figure 1.

\subsection{SOA-Based e-Government Architecture}

2.2.1. Mathematical Model of SOA Algorithm. The SOA algorithm directly simulates the intelligent behavior of human random search and is directly applied to the optimization problem solving. In the calculation process of the optimization solution, the intelligent search behavior can be understood as follows: in a search in a continuous space, generally better solutions may be in the vicinity of the better solution, and the optimal solution may be better solution. Therefore, if the searcher individual is in a relatively good position, the search scope should be set in a small space; if the searcher's location is not ideal, you should choose to search in a relatively large neighborhood. Therefore, when establishing a mathematical model for search criteria, SOA uses fuzzy logic that can effectively describe the language and uncertainty reasoning, and determines the step value of the search [7].

After a series of learning, the SOA algorithm gains relevant experience, combines the aggregation behavior of intelligent population spontaneous organization, the selfinterested self-priority behavior, and the pre-action behavior with predictive guidance ability, and finally establishes the individual. This is the direction of the search.

The indeterminate reasoning behavior in the SOA algorithm refers to the use of the approximation ability of the fuzzy system to simulate the intelligent search behavior of humans, and then to establish a bridge between behavior and cognition. By learning the intelligent random search behavior of human beings, we can use the fuzzy rules to describe the human search behavior: the smaller objective function corresponds to a smaller search step, and the search step is blurred [8].
Variables can usually be represented using Gaussian membership functions:

$$
\mu_{A}(x)=\exp \left[\frac{-(x-\mu)^{2}}{2 \delta^{2}}\right],
$$

where $\mu_{A}$ is Gaussian membership; $x$ is the input variable; $\mu$, $\delta$ are the membership function parameters.

If the value of the output variable is $[\mu-3 \delta, \mu+3 \delta]$ exceeded, and if $\mu_{A}(\mu+3 \delta)<0.0111$, its membership is negligible. So, you can set the value of the minimum membership to $\mu$ min $=0.0111$.

To design a fuzzification system that can be used in a variety of optimization problems in the logic of uncertainty, you need to sort the objective function values according to certain decrement rules and then convert the real-type function values into natural numbers. Regarding the integer value of the total number of populations $S$, the result of the transformation is used as the input value of the uncertainty logic. In order to make the ordering of the function values directly proportional to the membership degree, usually the maximum membership value is $\mu \max =1.0$ in the best position, so that the minimum membership degree is $\mu$ min $=0.0111$ in the worst position, and the rest position $\mu<1.0$ can pass formulas (2) and (3) for indication:

$$
\mu_{i}=\mu \max -\frac{s-I_{i}}{s-I}(\mu \max -\mu \min ), \quad i=1,2,3, \ldots, s,
$$

$$
\mu_{i j}=\operatorname{rand}\left(\mu_{i}, 1\right), \quad j=1,2, \ldots, D .
$$

In formulas (2), (3), $i$ is defined as the value of the objective function; that is, $\mu_{i}$ is the membership degree of $i$; the search space is set to $J$ dimension, the membership value $\mu_{i j}$ of $i$ in the space is $I ; I$ is the function value of the population. The descending order of size is the order value of $x(t)$ at this time; $D$ is the largest dimension in the space. In equation (3), $\mu_{i}, 1$ is a random real number in $\operatorname{rand}\left(\mu_{i}, 1\right)$. The condition through uncertainty reasoning, that is, equations (2) and (3), can be used to determine the membership value from the latter, and then according to the behavior part of the uncertainty reasoning.

Equation (4) gives the step size:

$$
a_{i j}=\delta_{i j} \sqrt{-\ln \left(\mu_{i j}\right)}
$$

In equation (4), the parameter value of the Gaussian membership function is defined as the search step value of the $J$-dimensional search space, and the value can be determined byequations (5) and (6):

$$
\begin{aligned}
\delta_{i j} & =w \cdot \operatorname{abs}\left(\vec{x}_{\text {min }}-\vec{x}_{\max }\right), \\
w & =\frac{T_{\max }-t}{T_{\max }} .
\end{aligned}
$$

In equations (5) and (6), the position of the maximum $\vec{x}_{\min }$ and minimum function values $\vec{x}_{\max }$ in the same subgroup are set to, and defined as, the inertia weight, and 


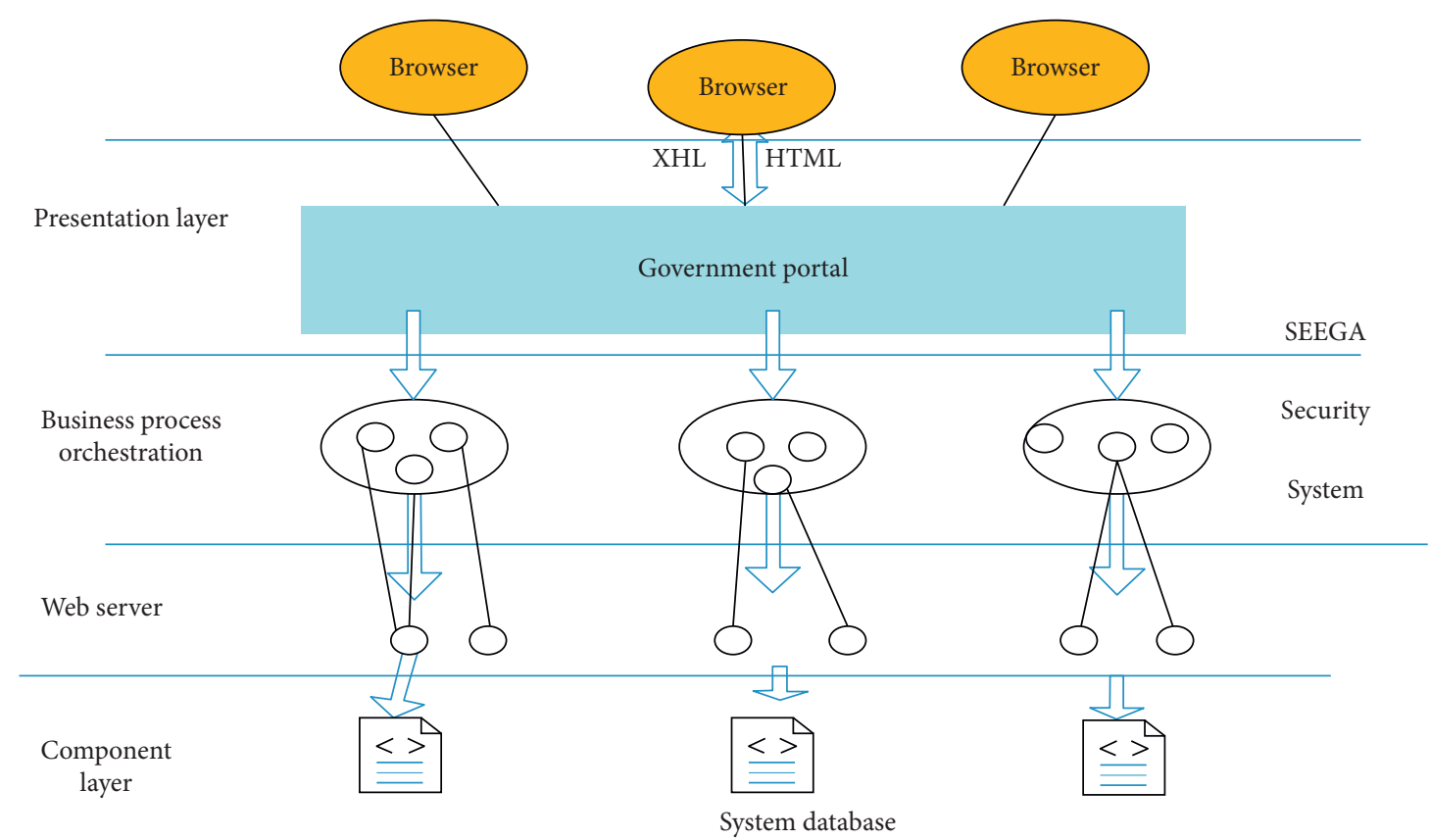

FIGURE 1: SOA based on the government system architecture.

their value linearly decreases with increasing the number of evolutions, and the decreasing interval is [0.1], 0.9]; and $t$ represents the maximum number of iterations, and $T_{\max }$ represents the current number of iterations in turn; abs ( ) represents the absolute value of each input value.

2.2.2. Layers of SOA-Based e-Government Architecture. The SOA-based e-government architecture consists of the following layers:

(1) Data layer: the data layer is simply a popular database management system for storing various system parameters and official documents such as official documents used in the government system; it can also be a data set of legacy systems.

(2) Component layer: this layer consists of the enterprise components responsible for implementing the functionality. These special components are a managed and controlled collection of enterprise assets supported at the enterprise and business unit level. In most cases, this layer uses container-based technologies such as application servers that implement components, load balancing, high availability, and workload management [9].

(3) Service layer: the services selected by the business to support and disclose are at this level. They can be discovered or directly statically bound, then called, or, if possible, orchestrated into a composite service. The service disclosure layer also provides mechanisms for acquiring enterprise-wide components, business unit-specific components, and, in some cases, specific project formations and materializing their interface subsets in the form of service descriptions. Therefore, enterprise components use the functionality provided by their interfaces to provide service implementations at runtime. The interface at this level is exposed as a service description, in which they are exposed for use. They can exist independently or as a composite service.

(4) Business process orchestration layer: the composition and orchestration of services exposed in the service layer are defined in this layer. By coordinating and orchestrating, services are bundled into a single process and thus act together as a single application. These applications support special use cases and business processes [10].

(5) Presentation layer: in the framework of the presentation layer, the form of the presentation layer is mainly the government portal, and the user accesses the business process and specific functions of the government system through the government portal.

(6) Enterprise service bus (ESB): this layer enables services to be integrated and is often described as ESB. Web services introduce a set of reliable performance sets such as intelligent routing, protocol mediation, and other transformation mechanisms. The Description Language (WSDL) defines a binding that contains the address of the service. On the other hand, ESB provides a location-independent mechanism for integration [8].

(7) Security system: the security system uses the current relatively complete security technology to ensure the security of the entire government system, such as SSL and XML encryption, to ensure the security of the transport layer and the security of information exchange. 
2.3. Using XML to Describe Data. According to the analysis in the previous section, in order to be able to realize the information interaction between the services in SBEGA, this information must be organized by an open, long-lived and widely accepted standard, and XML meets the above requirements. Data description specification, in SBEGA XML, will be used to describe the data that interacts between services. [11].

The Extensible Markup Language (XML) standard is a markup language for the text-based World Wide Web Consortium (W3 C) specification. Unlike HTML, which uses tags to describe appearance and data, XML strictly defines portable structured data. XML has some revolutionary features, making it the most fundamental and important technology in dynamic e-commerce. They are explained as follows:

(1) Scalable: the concept of XML is simple, but it has a wide range of applications, because anyone can define the corresponding XML tags to express the structure and type of information according to the needs of their own applications.

(2) Self-description: XML is a meta-language that can be interpreted and constrained by the definition of another XML document through an XML (previously through DTD, now XML Schema) definition.

(3) Information interoperability between heterogeneous systems: the XML format is simple to read and can represent any document material. As shown in Figure 1, as long as the system participating in the information exchange is equipped with an XML parser, it can interpret the information transmitted by other machines, thereby enabling communication of heterogeneous systems. Electronic Data Interchange (EDI) is a standard for electronic business documents, but it requires expensive software and a special network that is daunting for most companies. In contrast, XML-related software can be easily obtained, and it can use inexpensive, universal Internet transmission. [12]. The use of XML and the transmission of information and information will bring a revolutionary impact to e-commerce. XML transmission in heterogeneous systems is shown in Figure 2.

\section{Construction of Data Platform Based on Brainlike Computing}

3.1. Communication Mechanism Using SOAP Communication Protocol. The Simple Object Access Protocol (SOAP) is a lightweight, simple, XML-based protocol designed to interact with structured and solidified information on the Web. It can be used in conjunction with many existing Internet protocols and formats, including Hypertext Transfer Protocol (HTTP), Simple Mail Transfer Protocol (SMTP), and Multipurpose Internet Mail Extensions (MIME). It is independent of the underlying transport protocol, and the characteristics of adopting XML structured information are in line with the characteristics that SBEGA services may be heterogeneous, and interactive information is described by XML, which makes SOAP the first choice for communication protocols in SBEGA [13].

SOAP consists of four parts:

(1) SOAP encapsulation (envelop): encapsulation defines a framework that describes what is in the message, who sent it, who should accept it, and how to handle it.

(2) SOAP encoding rules are used to represent instances of the data types that the application needs to use.

(3) SOAP RPC representation is an agreement representing remote procedure calls and responses.

(4) SOAP bindings use the underlying protocol to exchange information.

Although these four parts are defined as a part of SOAP in a holistic manner, they are functionally intersecting, independent, and special. Envelope and encoding rules are defined in different XML namespaces. This makes the definition simpler. All SOAP information is an XML document. It must have a SOAP envelope and an information body, as well as an optional SOAP header. As shown, each element has a corresponding set of special rules. SOAP application system client and server communication model is shown in Figure 3.

3.2. SBEGA Service Mechanism. Web services are a new generation of Web applications, a type of software component that can be accessed through open Internet protocols. They represent a combination of component technology and Web technology. The traditional services based on CORBA, DCOM, and other proprietary protocols obviously do not conform to the openness of SBEGA architecture. The web services standard has been recognized by major platform providers and system service providers and is becoming the de facto service implementation standard. The use of web services to implement services in SBEGA is a natural choice [14].

In the web services invocation framework, clients and services communicate via standard Web protocols (HTTP or SMTP) and XML, which are completely platform-independent and implementation-oriented. Web services solve the main problems of government's government activities through the Internet, such as interoperability and integration. At the same time, web services have open and easy-to-implement standards, which enable various heterogeneous legacy applications within the government to be integrated. The architecture of web services is a layered structure, which can be divided into network layer, message delivery layer, service description layer, service discovery layer, and workflow layer. Its security, management, and quality of service run through the entire architecture of the web service [15].

The network transport layer is the basis of the Web protocol stack. To be called by the service requester, the web services must pass through the network. The standard network protocol used by web services in the Internet environment is generally HTTP, and SMTP and FTP protocols are also supported in some extension areas. 


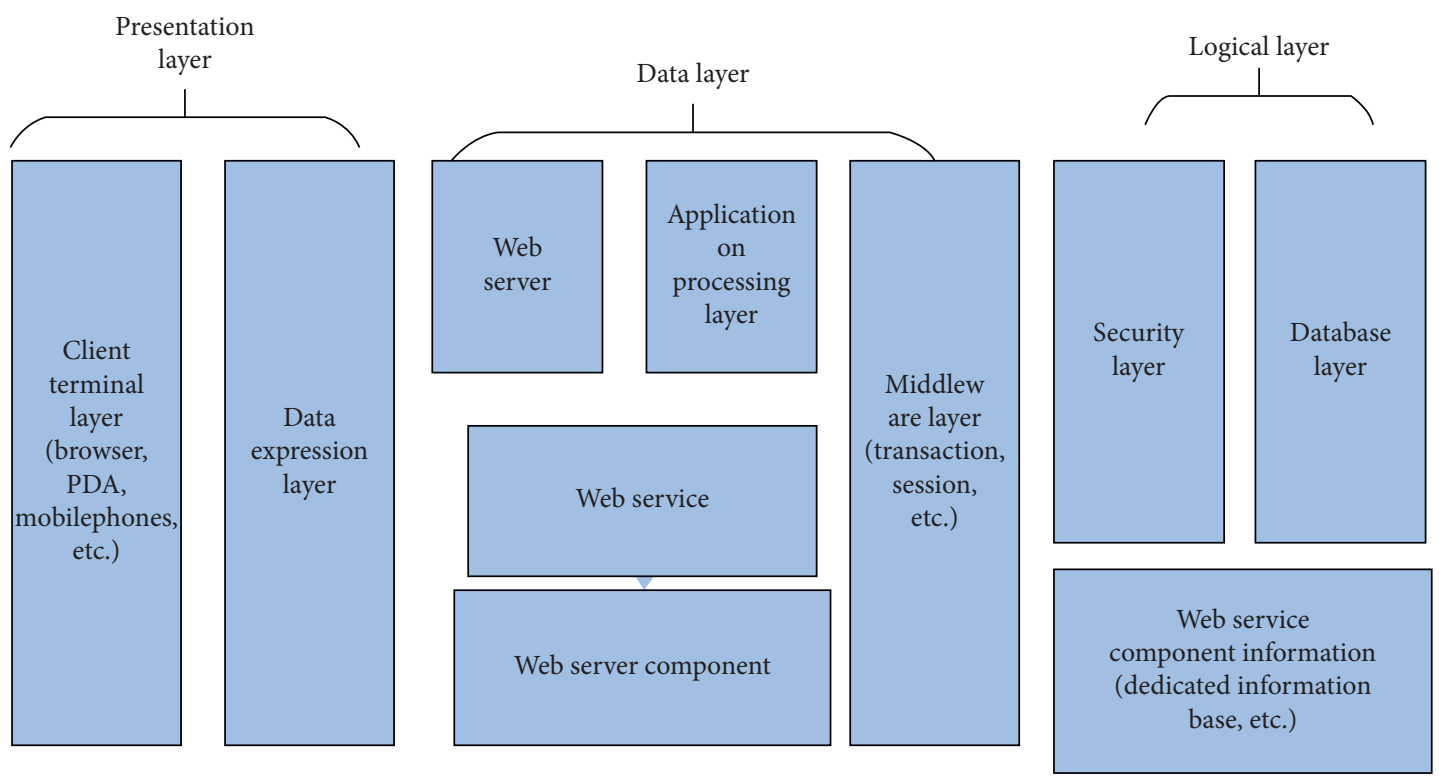

FIgURE 2: XML transmission in heterogeneous systems.

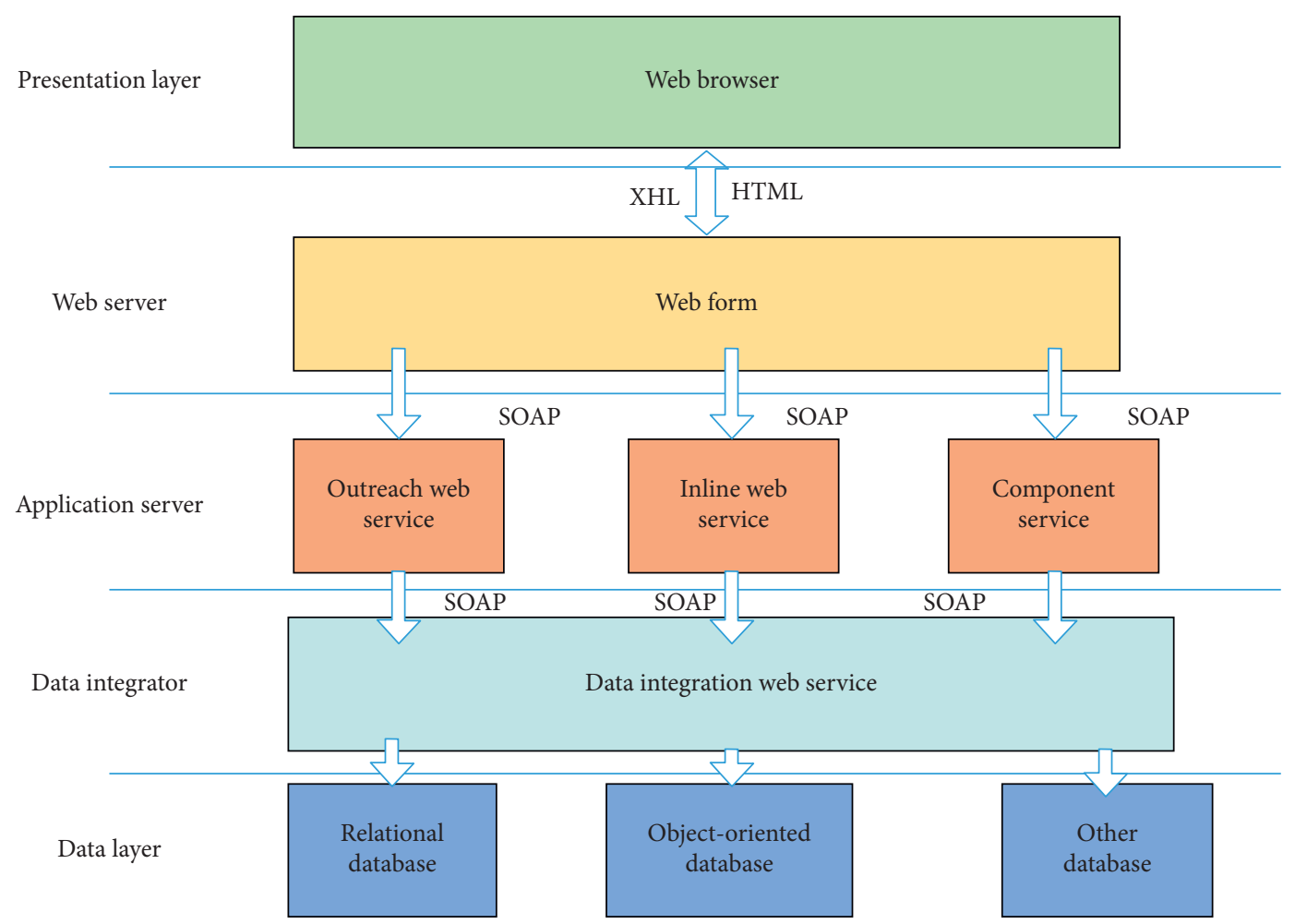

FIgURE 3: SOAP application system client and server communication model.

The message service layer uses the XML-based messaging protocol SOAP, which is built on top of the lower transport layer, which means that SOAP can be used alone or in conjunction with any transport protocol. All SOAP messages support the web services architecture, operations such as publishing, binding, and finding.

3.2.1. Service Description Layer. WSDL is a basic standard for defining the implementation and interface of services based on XML format. That is to say, WSDL divides the description of the service into two parts: the service implementation and the service interface.

3.2.2. Service Publishing Layer. The service provider can send the WSDL document directly to the service client, and the service provider can also publish the WSDL document to a local WSDL registry, or a public/private UDDI registry. Service clients can use these registration libraries to obtain 
WSDL documents. UDDI provides a common set of SOAP APIs that implement a service registration method. The UDDI specification facilitates the creation, description, discovery, and integration of web services.

3.2.3. Service Discovery Layer. Service discovery is based on service release. If web services do not or cannot be published, then they cannot be discovered.

3.2.4. Service Workflow Layer. Web services Workflow Language (WSFL) is the standard for the service workflow layer at the top of the protocol stack. Unlike other standards in the protocol stack, WSFL is aimed at business process modeling and workflow.

3.3. SBEGA's Safety System. Through the security requirements of SBEGA, as well as the analysis of transport layer security and information interaction security, it can be seen that the transport layer security and XML security meet the different security requirements of the system, as shown in Table 1. The most important part of SBEGA's security system is to ensure the security of information exchange and transmission between different services in the system. From the table, we can see that if one of the security technologies is used alone, the security requirements of such information cannot be guaranteed. Only by combining them according to their characteristics, SBEGA's security system is established, and the functions provided by the transport layer security and XML security are fully utilized to cooperate with each other to meet the security requirements of SBEGA.

The SBEGA security system combines transport layer security and XML security to achieve the security requirements for SOAP messages passed between services in SBEGA, as shown in Figure 3. The requester uses SSL or HTTP basic authentication combined with SSL to complete the sender's authentication and uses the session key determined by the SSL negotiation to send the encrypted message to the receiver, thus establishing security between the sender and the receiver. XML digital signatures and XML encryption work at the transport layer security to provide a higher level of data confidentiality and data integrity. The nonrepudiation of the message can be provided by combining sender authentication with SSL [14]. SBEGA's security system is shown in Figure 4.

\section{SBEGA Application in e-Government Platform}

Over time, governments often have multiple heterogeneous independent systems based on different platforms and architectures, operating in different functional departments. With the increasing number of interactive businesses of citizens, businesses, and governments in the future, government departments need to continuously expand their systems, which in the long run will bring great inconvenience to the maintenance of the system in the future. Moreover, the subsystems of various departments are not connected, and there is almost no way for them to cooperate with each other. Therefore, information islands are often formed within the government system. Therefore, to get rid of this situation and ensure decentralized information resources under the premise of ensuring information security, the unity, integration, and management have become a key to improving the application level of e-government. At the same time, with the changes in policies, the departmental integration and adjustment of government agencies are frequent, and the business processes of the government system need to be constantly changing in order to cope with such policy changes. Process reorganization is an extremely frequent matter and requires collaborative work between multiple departments [15]. Application systems between multiple departments must agree on message formats, communication protocols, data models, etc., using standardized docking methods, so as to effectively realize the interaction of dynamic e-government modes. This requires a unified, standard-based technology, SOA architecture can well meet this need, and the system can be divided into service components according to different functions, through the UDDI registry, the public portal and the backstage of each department. The service is bound and called. Therefore, this chapter will design an e-government approval system model based on the e-government platform based on SOA architecture to realize a "one-stop," flexible and scalable e-government approval system.

4.1. e-Government Approval System Architecture. From the various advantages of SOA mentioned above, from the perspective of business and IT, service-oriented architecture is an architecture that can well adapt and solve the current e-government malpractices. The use of this structure to build an e-government approval system can solve the "information island" in the e-government field. It is the interconnection and interoperability of subsystems of various departments, integrating existing partner systems, and the rapid changes of corresponding business services to meet the needs of the public. The architecture of the e-government approval system model for service-oriented architecture is shown in Figure 5 [16].

According to the network division of each part of the model, we describe the various parts of the model as follows.

4.1.1. External Network. The model in the external network mainly runs on the Internet, providing services to the public and government office workers through Internet access and Web technologies. The one-stop office platform is mainly for internal government personnel, providing the entrances of various departments to their respective approval interfaces. It can manage the approval process and realize online approval, joint approval, handling supervision, license printing, statistical analysis, data query, and information feedback. Wait for the energy, to achieve online "one-stop" service [17]. On the public network, you can carry out project consultation, application form download, online submission application, approval result release, online information exchange, identity authentication, online complaints, and other functions [18]. 
TABLE 1: Analysis of transport layer security and XML security.

\begin{tabular}{lcccc}
\hline Security requirements & SSL & XML encryption & XML digital signature & Firewall \\
\hline Confidentiality & Point-to-point confidentiality & End-to-end machine confidential & & \\
Authentication & Using MAC & Using MAC and digital sign & ID authentication & Name authentication \\
$\begin{array}{l}\text { Integrity } \\
\text { Authorization }\end{array}$ & & & Signature & Authorize \\
Nonrepudiation & & & Send/receive & \\
\hline
\end{tabular}

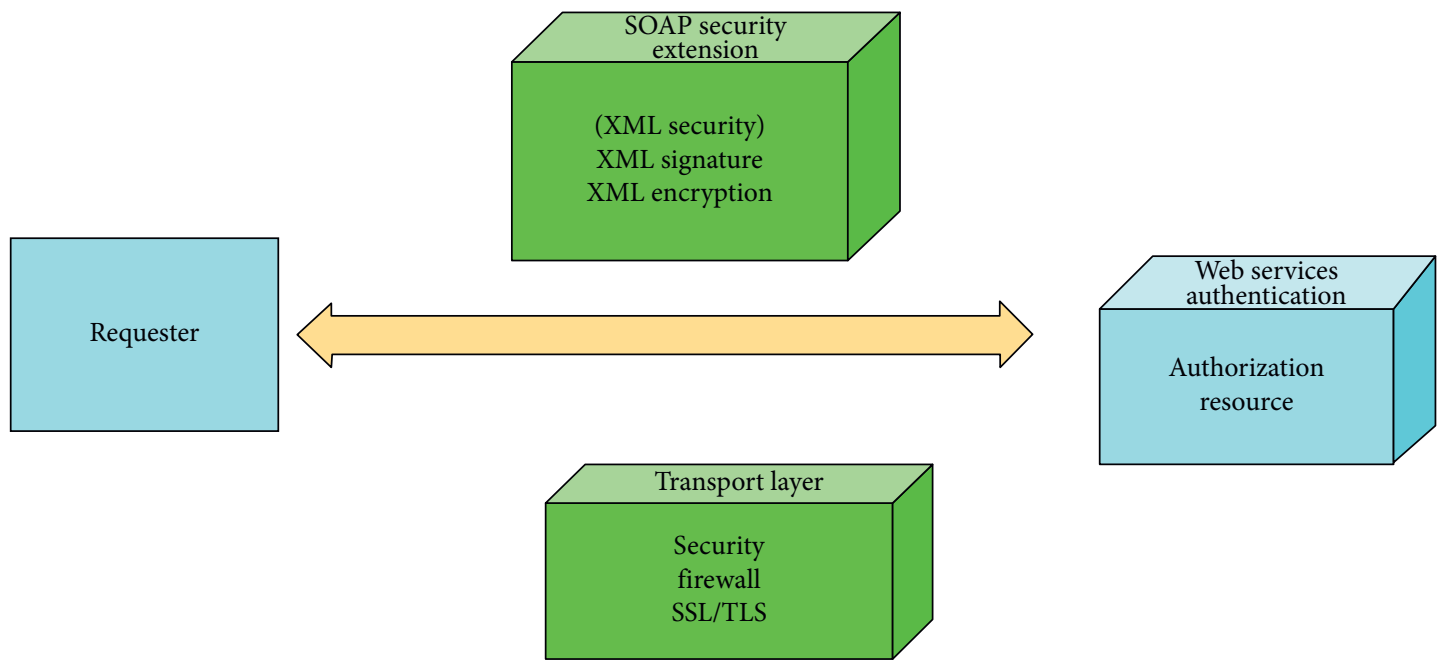

FIgURE 4: SBEGA's security system.

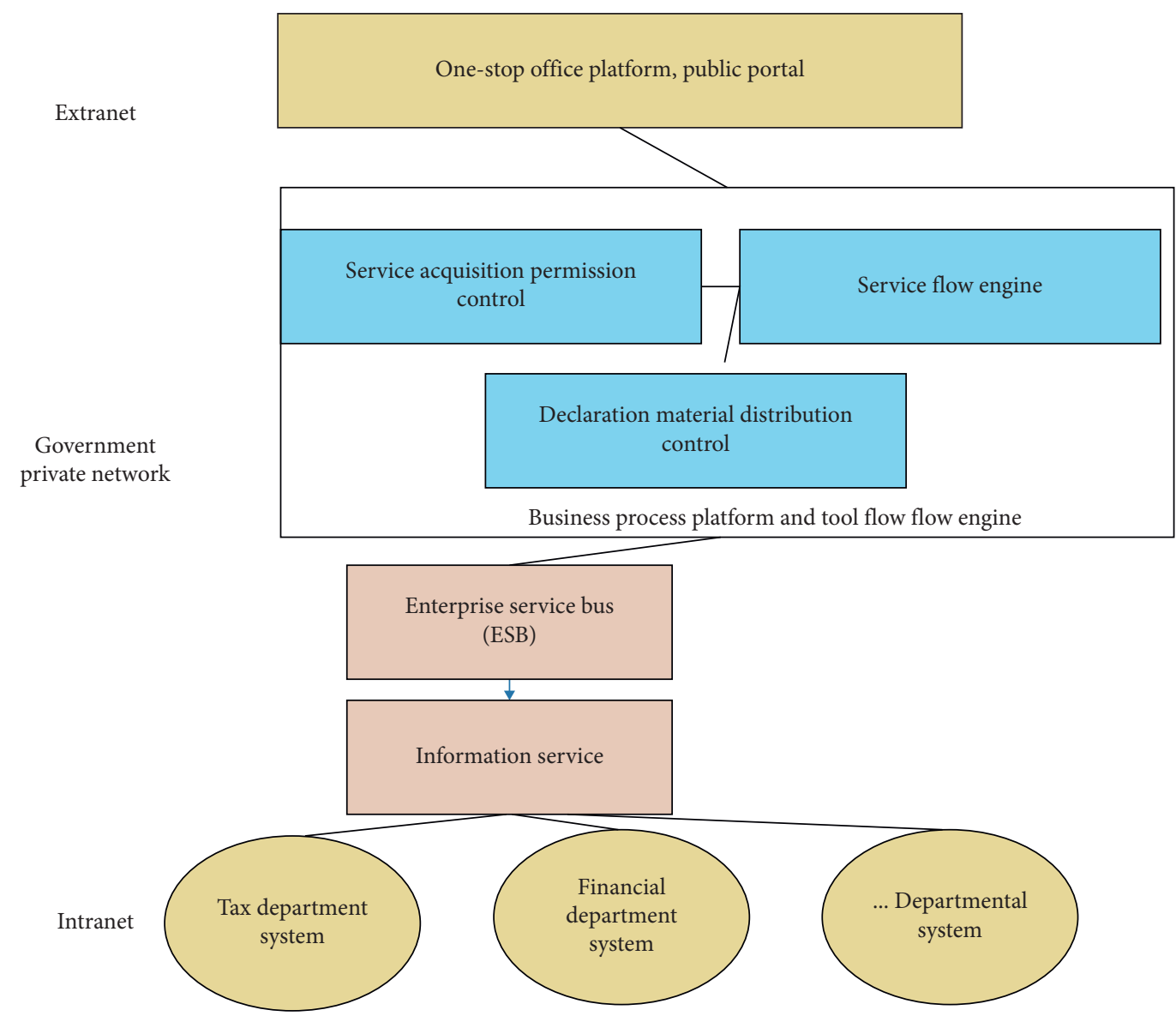

FIGURE 5: Architecture of the e-government approval system model for service-oriented architecture. 
4.1.2. Government Special Network. The enterprise applying for approval will register the identity through the public portal. This registration process will call to check whether the enterprise is legal. The service checks whether the enterprise has legal status. After the registration is successful, it enters the approval resource library, and the enterprise user submits all the application items. After the data, the service flow engine automatically distributes it to the systems of different approval departments for review based on the nature of the data. After the approval personnel of various government departments enter the unified office platform, the system automatically pushes the approval part of each department to use the event service to the individual's desk [19]. The approval results of each part are automatically stored in the approval resource database, and the system monitors the time limit for approval. If the timeout is to be exceeded, the calendar reminder service or the SMS notification service in the tool set is called to automatically remind the approver [14].

Each department registers its service description information in the government approval UDDI and obtains services through the search for services. Some services have certain confidentiality. Therefore, not all new systems can call the registered services arbitrarily. The service gets access to the new control component to get access to it.

4.1.3. Intranet. The subsystems of each department run on the intranet, package the shared functions of each subsystem into an information service, provide the required information externally, or build new web services according to requirements, and process the shared services. Orchestrate, build new applications, recreate new services when the approval requirements change, and reengineer the original process layout, thereby improving the software reuse rate and shortening the software delivery cycle.

4.2. System Design. From the analysis of the previous section, the functional requirements of the market detection and control office can be abstracted into statistical analysis of enterprise data reporting and reported data. In order to realize a flexible, loose and scalable e-government system, the SOA-based e-government system architecture (SBEGA) proposed in this paper is used to implement the case. The architecture of the case e-government system is shown in Figure 5. In the architecture shown, the main functional modules of the case system are as follows [15].

4.2.1. User Management Service. The service provides external system user management through the web services interface. It has two components: enterprise user management and department internal user management. Their respective functions are Enterprise User Management Components.
4.2.2. Data Reporting Service. The data reporting service provides data services to enterprises and also supports the exchange of data between the future case system and other government departments' e-government systems. In the future, if the e-government system of other government departments needs to transmit the enterprise report data to the case system, the data transmission can be performed by defining the SOAP message format that satisfies the service interface requirements and accessing the service.

The service consists of data verification, conflict checking, data storage processing, and other components. These components perform data validity verification on the data transmitted by the service interface, check whether it conflicts with historical data, and perform data storage processing.

4.2.3. Data Statistics Service. The data statistics service is a service provided by the internal management personnel to collect statistics on the data reported by the enterprise. Like the data reporting service, the data statistics service also supports the exchange of data with the e-government systems of other government departments in the future. In the future, if the e-government system of other government departments needs to obtain the statistical results reported by the enterprise from the case system, it only needs to access the standard interface provided by the service and parse the data returned by the interface according to the rules [20].

4.3. Service Implementation. The service is the core of SBEGA. This section starts from this core and takes the user management service in the case system as an example to introduce the implementation of the service in the e-government system built by SBEGA. At the end, the remote access and function replacement of the service are carried out.

4.3.1. Service Realization. The case system uses the open source organization Axis 2 developed by Apache as the SOAP server running by web services. Axis 2 is newly written based on the new architecture and does not use the common code of Axis 1. Compared with Axis 1, it is more modular, more flexible, and more efficient in architecture. Using Axis 2 as the running platform of web services effectively reduces the difficulty of web services development and deployment. The process of developing web service based on Axis 2 is as follows [12].

(a) Write Service Core Business Implementation. In SBEGA, the service is the interface for the core business process to be developed externally. Take the user management service as an example. The core business is to add, update, and query enterprise users and internal users. To complete these services, many components and classes may be involved. However, users do not want to open all interfaces as services. In order to maintain the future maintainability of the system, 
we design a special service class, in which all operations are open to the service, as shown in the code snippet above.

Package com.seagull.services;

Import"'”;

Public class UserManage \{

Public OMElement adduser (OMElement userdata) \{

Return resp;

Public OMElement updateuser (OMElement userdata) \{

Return resp;

Public OMElement getuser (OMElement searchdata) \{

Return resp;

(b) Write a Deployment Descriptor. After the core business is implemented, it is published as a service to the Axis 2 server, and a deployment description must be written for these implementations. The deployment descriptor is a standard XML file, and the above user-managed deployment descriptor document service.xml is as follows.

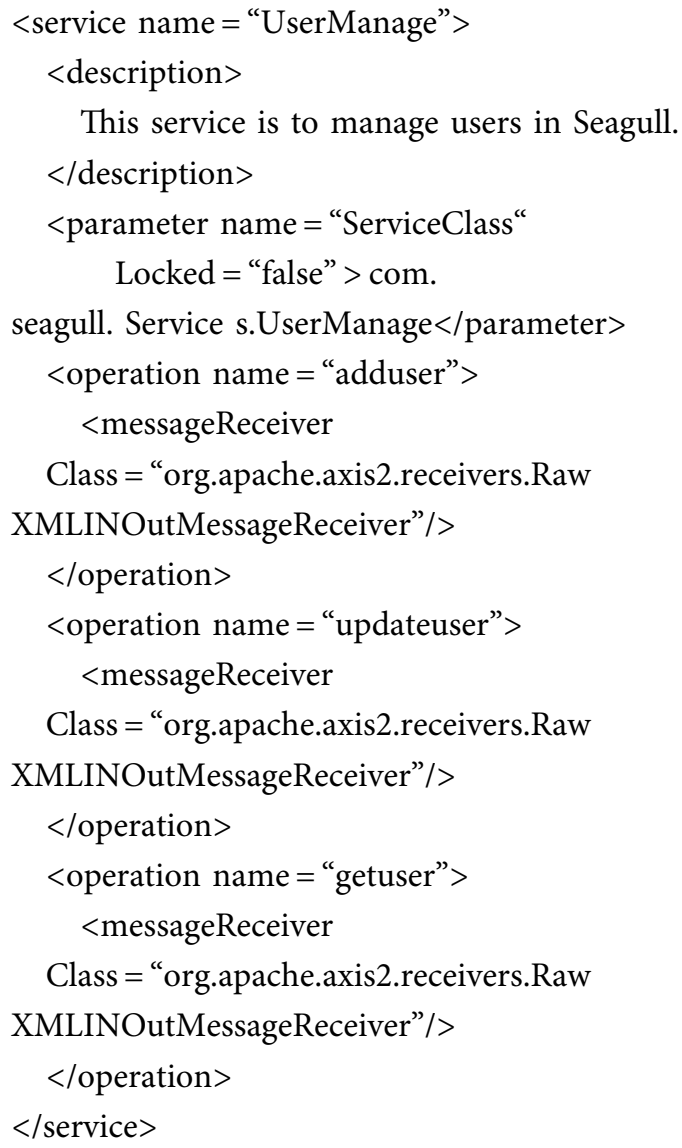

4.3.2. Package Service. According to Apache's specifications, the Axis 2 service is packaged as Axis Archive (.aar). This is a JAR file (created using the $\}$ ar or zip program), and the deployment descriptor document services.xml file is packaged in the 1VIETA-INF directory of this JAR archive.

4.3.3. Deployment Services. Deploying services in Axis 2 is as simple as copying the as: file into the axis2/WEB-INF/ services directory in the axis container's axis 2 web application. Another way to deploy a service is to use the Upload Service tool in the Axis 2 Management Console. Access the Axis 2 management console via http://:// localhost: <port $\} /$ axis2 and go to the administration page via the Administration link. Enter your username and password to $\log$ in. In the Tools section, select the Upload Service link, select the service to be deployed, package the .aar file, and then click Upload to complete the service deployment. This method is used to open source services on remote Axis 2 servers.

4.4. Government Affairs Platform Simulation Experiment. Public value evaluation and prediction: based on the above discovery model, customer value evaluation and customer prediction are conducted. First, the average model value of each type of customer is calculated as the classification basis of the four types of customer. The predicted customer and average model values are calculated in a fuzzy way to achieve the prediction purpose. In the government affairs system, the relevant information of customers is extracted at will and converted into data according to quantitative standards, as shown in Table 2. The distribution of customer information is shown in Figure 6.

Take the customer classification model of $\lambda=0.40$ and find the average index of each model, as shown in Table 3. The average index distribution of customer value is shown in Figure 7.

Comparing the current value and potential value of customers, it can be seen that the potential value of bank customers finally converting into the value of return value is an uncertain amount. Therefore, the selection of customer value should be more focused on the current value, so this paper selects the value after the current value is added as the basis for clustering customer value. After addition, Class $A$ customers are high-value customers, Class $D$ customers are potential value customers, Class $C$ customers are general value customers, and Class $B$ customers are low-value customers. 
TABLE 2: Customer information.

\begin{tabular}{|c|c|c|c|c|c|c|c|c|c|c|}
\hline $\begin{array}{l}\text { Classification } \\
\text { object }\end{array}$ & Overdraft & $\begin{array}{l}\text { Feedback } \\
\text { personnel }\end{array}$ & $\begin{array}{l}\text { Invalid } \\
\text { feedback }\end{array}$ & $\begin{array}{c}\text { Overall } \\
\text { customer } \\
\text { status }\end{array}$ & $\begin{array}{c}\text { Personal } \\
\text { response } \\
\text { fact }\end{array}$ & $\begin{array}{c}\text { Customer } \\
\text { recommendation } \\
\text { number }\end{array}$ & $\begin{array}{l}\text { Number } \\
\text { of replies }\end{array}$ & $\begin{array}{c}\text { Random } \\
\text { testing }\end{array}$ & $\begin{array}{c}\text { The } \\
\text { number of } \\
\text { responses }\end{array}$ & $\begin{array}{c}\text { Required and } \\
\text { unpurchased } \\
\text { business volume }\end{array}$ \\
\hline $\begin{array}{l}\text { Forecast } \\
\text { customers }\end{array}$ & 2015 & 25630 & 200 & 48 & 50 & 6 & 56000 & 75633 & 5000 & 7 \\
\hline
\end{tabular}

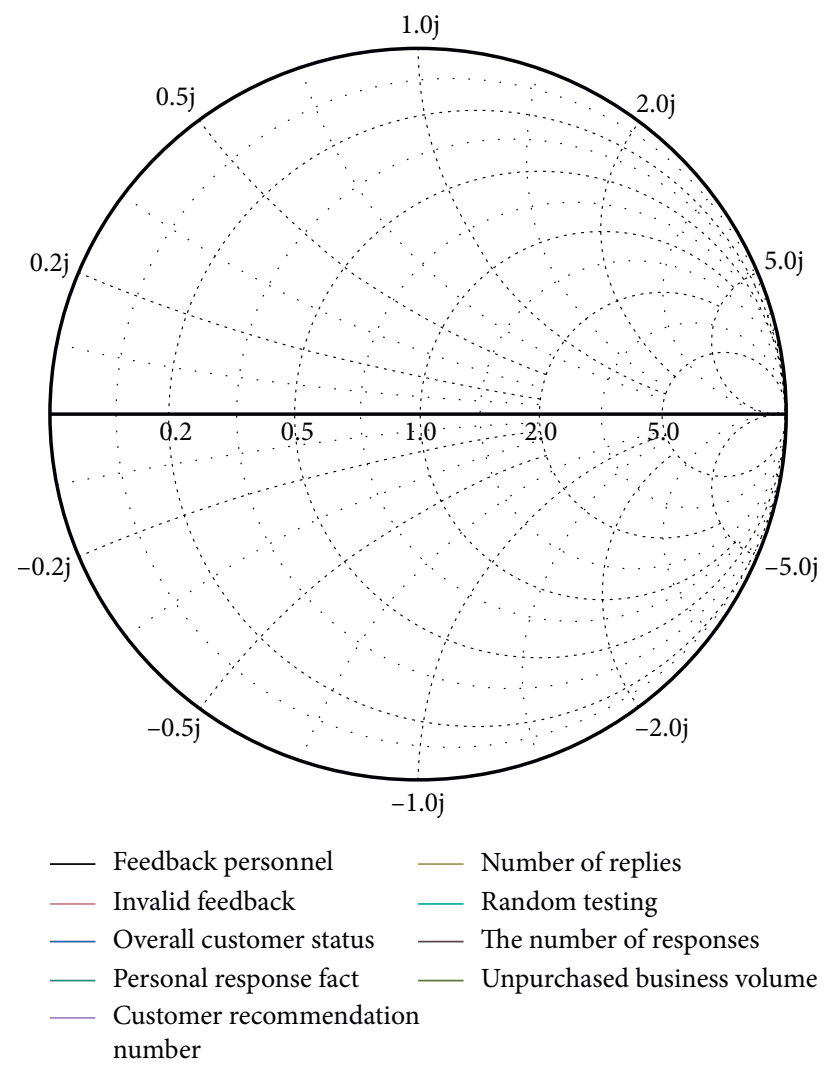

FIGURE 6: Customer information distribution map.

TABLE 3: Average indicator of customer value.

\begin{tabular}{|c|c|c|c|c|c|c|c|c|c|c|}
\hline $\begin{array}{l}\text { Classification } \\
\text { object }\end{array}$ & Overdraft & $\begin{array}{l}\text { Feedback } \\
\text { personnel }\end{array}$ & $\begin{array}{c}\text { Invalid } \\
\text { feedback }\end{array}$ & $\begin{array}{c}\text { Overall } \\
\text { customer } \\
\text { status }\end{array}$ & $\begin{array}{l}\text { Personal } \\
\text { response } \\
\text { fact }\end{array}$ & $\begin{array}{c}\text { Customer } \\
\text { recommendation } \\
\text { number }\end{array}$ & $\begin{array}{l}\text { Number } \\
\text { of replies }\end{array}$ & $\begin{array}{c}\text { Random } \\
\text { testing }\end{array}$ & $\begin{array}{l}\text { The } \\
\text { number of } \\
\text { responses }\end{array}$ & $\begin{array}{c}\text { Required and } \\
\text { unpurchased } \\
\text { business volume }\end{array}$ \\
\hline $\begin{array}{l}A \text { class } \\
\text { customer }\end{array}$ & 3001 & 16211 & 18 & 83 & 50 & 9 & 150000 & 200000 & 30000 & 2 \\
\hline $\begin{array}{l}B \text { class } \\
\text { customer }\end{array}$ & 43567 & 0 & 9673 & 47 & 20 & 4 & 60000 & 3000 & 60000 & 1 \\
\hline $\begin{array}{l}C \text { class } \\
\text { customer }\end{array}$ & 411 & 67327.50 & 27980 & 56.5 & 20 & 22.5 & 26732 & 2716.5 & 100000 & 3 \\
\hline $\begin{array}{l}D \text { class } \\
\text { customer }\end{array}$ & 3267.65 & 41786.42 & 2788.58 & 54 & 41.15 & 4.42 & 117368.65 & 15739.35 & 63076.92 & 2.85 \\
\hline
\end{tabular}




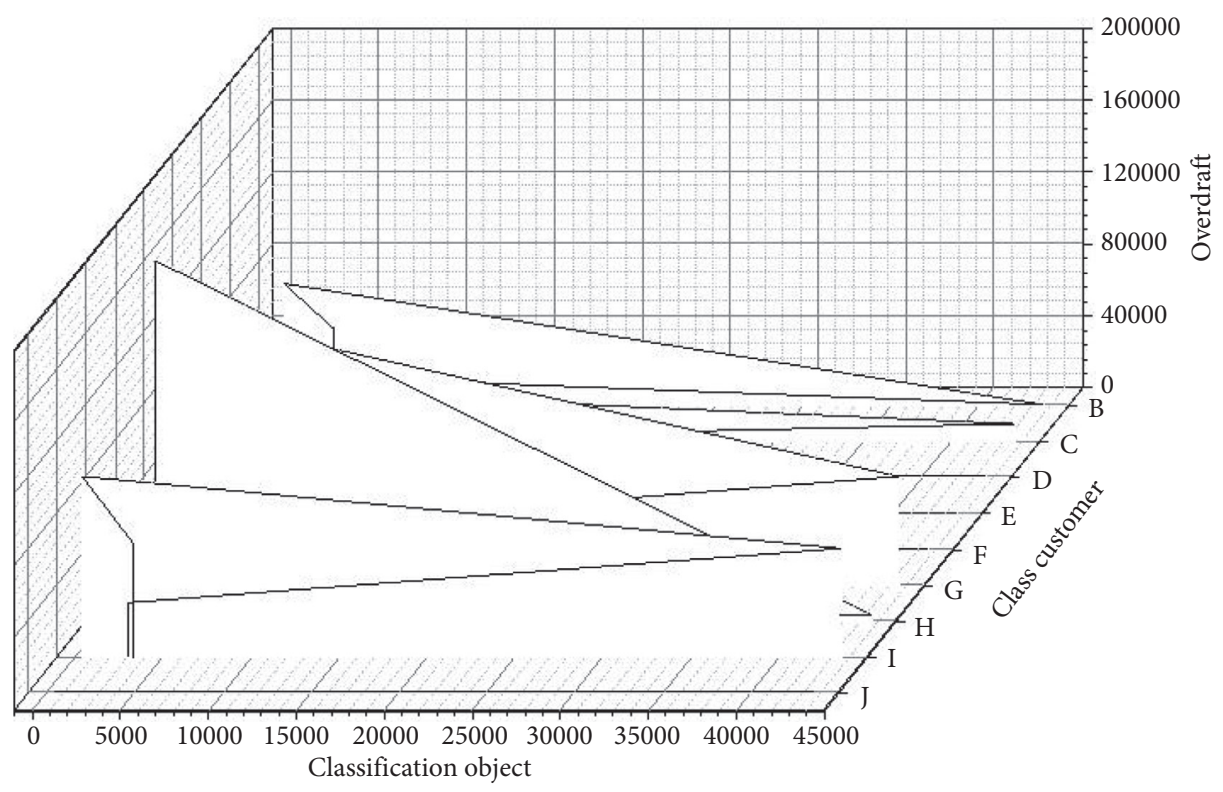

Figure 7: Average indicator distribution of customer value.

\section{Conclusion}

Big data is considered a diverse form of unstructured data, which need proper analysis and management, and it is excessive technological revolution after the Internet, the Internet of Things, and cloud computing. This paper analyzes the current popular SOA and web services technologies, illustrates the feasibility of their use in e-government systems, and proposes SOA-Based e-Government Architecture (SBEGA). SBEGA's basic mechanism, like other information systems, e-government networks, and information, is also subject to security threats. SBEGA uses an open architecture, which is subject to more security threats. This paper analyzes SBEGA's security objectives. The security system of SBEGA was put forward. Finally, SBEGA was successfully applied to the actual case e-government system, focusing on the system architecture design and representative service realization of the case, and testing the scalability of the case. Through the practice of the case system, SBEGA can meet the requirements of the current e-government system's openness and interconnection and provide a safe and flexible system architecture for building a dynamic and scalable e-government system, for the future construction of similar e-government systems, with reference and promotion value.

\section{Data Availability}

The datasets used and/or analyzed during the current study are available from the corresponding author upon reasonable request.

\section{Conflicts of Interest}

The authors declare that they have no conflicts of interest.

\section{References}

[1] L. Ying, "Design and development of public affairs government management system based on Internet," $A d$ vanced Materials Research, vol. 9, no. 12, pp. 1552-1555, 2014.

[2] L. Xuesi, "Based on the experience of E-government affairs how to design electronic port in China," Applied Mechanics and Materials, vol. 6, no. 3, pp. 1539-1542, 2014.

[3] W. Xingbiao and Y. Yiming, "Research on standardized management in government affairs microblog," Enterprise Science and Technology and Development, vol. 8, no. 8, pp. 859-733, 2013.

[4] J. Li, Z. Zheng, Q. Tian, G. Zhang, F. Zheng, and Y. Pan, "Research on tridiagonal matrix solver design based on a combination of processors," Computers and Electrical Engineering, vol. 62, pp. 1-16, 2017.

[5] X. Li, Z. Lv, Z. Zheng, C. Zhong, I. H. Hijazi, and S. Cheng, "Assessment of lively street network based on geographic information system and space syntax," Multimedia Tools and Applications, vol. 76, no. 17, pp. 17801-17819, 2017.

[6] S.-X. Pan, W.-J. Sun, and Z. Zheng, "Video segmentation algorithm based on superpixel link weight model," Multimedia Tools and Applications, vol. 76, no. 19, pp. 19741-19760, 2017.

[7] M. Chahal, S. Harit, K. K. Mishra, A. K. Sangaiah, and Z. Zheng, "A Survey on software-defined networking in vehicular ad hoc networks: challenges, applications and use cases," Sustainable Cities and Society, vol. 35, no. 11, pp. 830-840, 2017.

[8] T. Yang, X. Long, A. K. Sangaiah, Z. Zheng, and C. Tong, "Deep detection network for real-life traffic sign in vehicular networks," Computer Networks, vol. 136, no. 8, pp. 95-104, 2018.

[9] Y. Sun, C. Li, G. Li et al., "Gesture recognition based on Kinect and sEMG signal fusion," Mobile Networks and Applications, vol. 23, no. 4, pp. 797-805, 2018.

[10] C. Hamer, "Reinforcing the NPT," Australian Journal of International Affairs, vol. 65, no. 5, pp. 578-589, 2011. 
[11] C. Li-Jun, C. T. Co, and L. Y. Branch, "The design exploration of the government affairs cloud platform based on trusted computing at province class," Software Guide, 2018.

[12] L. I. Cheng-Lin and L. Liang, "Research and implementation of government affairs cooperation cloud platform based on electronic handwriting signature and mobile security," Communications Technology, 2018.

[13] J. Yiwei, "Information sharing platform in electronic government affairs," Computer and Digital Engineering, vol. 116, no. 1, pp. 143-167, 2009.

[14] C. Bingbing, "MOOCS curriculum model: contribution and dilemma," Foreign language Audio-Visual Teaching, no. 3, pp. 39-40, 2014.

[15] B. Yingcai, "A probe into the reform of college english teaching in art colleges and universities under the background of mousing course," Journal of Kaifeng Institute of Education, no. 9 , pp. 41-42, 2015

[16] Y. U. Miao, W. Yan-Zhang, and L. Ji-Shan, "Design on E-government affairs system platform supporting governmental affair process reconfigure," Computer Integrated Manufacturing Systems, vol. 10, no. 3, pp. 352-358, 2004.

[17] S. Shivani, S. Tiwari, K. K. Mishra, Z. Zheng, and A. K. Sangaiah, "Providing security and privacy to huge and vulnerable songs repository using visual cryptography," Multimedia Tools and Applications, vol. 77, no. 9, pp. 11101-11120, 2017.

[18] N. Kumar, S. Tiwari, Z. Zheng, K. Krishn, and A. Kumar Sangaiah, "An efficient and provably secure time-limited key management scheme for outsourced data," in Concurrency and Computation: Practice and Experience, Wiley, New York, NY, USA, 2018.

[19] M. Carman, M. Carman, N. Bonalumi et al., "ENA health care reform platform," Journal of Emergency Nursing, vol. 34, no. 5, pp. 392-393, 2008.

[20] R. C. Joseph and N. A. Johnson, "Big data and transformational government," It Professional, vol. 15, no. 6, pp. 43-48, 2013. 